\section{P234 PAEDIATRIC AND EMERGENCY STAFF ADHERENCE TO DKA GUIDELINES IN A PERIPHERAL PAEDIATRIC CENTRE}

Beth Gordon*, Pankaj Agrawa, Laura Crowley, Conor Cronin, Stephen MP O'Riordan. Paediatric Department, Cork University Hospital, Cork, Ireland

\subsection{6/archdischild-2019-epa.584}

Background Cork University Hospital (CUH) is peripheral centre of paediatrics that provides a joint adult and paediatric emergency department service. Currently paediatric and emergency department staff use the 2009 BSPED Diabetic KetoAcidosis (DKA) protocol. In light of upcoming new national DKA guidelines used by the Tertiary Paediatric centres, we aimed to see staff adherence to the current protocol in use.

Objectives To evaluate paediatric \& ED staff adherence to this protocol through documentation of management. Our secondary objectives included analysis of patient demographics, severity of DKA, complications and length of stay.

Methods We retrospectively analysed all paediatric patients with T1DM presenting with DKA from January 2015 until July 2017. Patients were identified from Diabetes Nurse Specialist database. Data was recorded and comparison was made between initial treatment from emergency staff and subsequent review by paediatrics.

Results The total number of patients on the DKA protocol during the time period was 53 and, following exclusion criteria, 37 (69\%) charts were analysed. Notable exclusions were 6 patients (11\%) commenced on protocol despite not meeting criteria for DKA diagnosis.

The average age at presentation was 8.18 years, male to female ratio $2: 3$ and $81 \%(n=43)$ of presentations were new onset T1DM. A majority (56\%) of presentations were of moderate severity.

Within management from ED staff, 28\% $(n=10)$ had no protocol documentation.

Only $8 \%(n=3)$ had the correct protocol documented and commenced. $31 \% \quad(n=11)$ were seen by paediatric team directly. Paediatric staff incorrectly calculated protocol management plans in $65 \%(n=24)$ of cases.

Despite $55 \%$ of presentations in severe acidosis meeting protocol requirement for ICU involvement they were not reviewed by the ICU team and ward managed.

Conclusion Health care providers working in CUH adherence to BSPED 2009 guidelines protocol is poor. It is essential to implement the use of current DKA protocol in use for all DKA at presentation. Recognition of the definition is the starting point.

We recommend thorough emergency $\&$ paediatric staff education and implementation of a mandatory proforma for DKA presentations.

\section{P235 THE PREVALENCE OF MICROALBUMINURIA IN CHILDREN WITH TYPE 2 DIABETES MELLITUS (T2DM)}

Michael Yafi*. UTHealth, The University of Texas Health Science Center at Houston, Houston, USA

\subsection{6/archdischild-2019-epa.585}

Background The prevalence of childhood-onset type 2 diabetes mellitus (T2DM) has increased globally, reflecting the pediatric epidemic of overweight and obesity. Children with T2DM have a higher prevalence of other comorbidities at diagnosis, including obesity, elevated blood pressure, dyslipidemia, fatty liver disease and microalbuminuria, compared to youth with type 1 diabetes of a similar age.

Method A retrospective chart review of the last twenty five children with documented diagnosis of Type 2 diabetes was performed

Results Patients ranged in age from 8 to 15 years at diagnosis. The female:male ratio was 1.3:1. Sixty percent of patients were Hispanics. All BMIs were above $85^{\text {th }}$ percentile for age and sex. Twelve percent of them had micro or macroalbuminuria at diagnosis (only $60 \%$ had persistent microalbuminuria after follow up) while only $5 \%$ had documented persistent hypertension.

Conclusion Screening for renal disease in children with T2DM should begin at diagnosis and annually thereafter. The best recommended test is a first-morning or random urine for albumin to creatinine ratio. Careful laboratory documentation of persistent albuminuria is critical since transient albuminuria can be caused by acute hyperglycemia at diagnosis. It is therefore important to demonstrate persistent albuminuria over a three- to six month period. Persistent albuminuria is defined as an elevated urine albumin to creatinine ratio in two of three urine samples taken over three to six months.

The diagnosis of T2DM in young children raises concern regarding the possibility of related comorbidities including renal involvement as a similar concern of adult presentation. Early diagnosis, monitoring and treatment of microalbuminuria can prevent the progression of renal disease in diabetes.

\section{P236 PARENTS' EXPERIENCES OF HAVING A CHILD DIAGNOSED WITH TYPE 1 DIABETES: INITIAL IMPACT AND CHANGE OVER TIME}

${ }^{1}$ Caroline Rawdon*, ${ }^{1}$ Pamela Gallagher, ${ }^{2}$ Michele Glacken, ${ }^{3,4}$ Nuala Murphy, ${ }^{5}$ Veronica Swallow, ${ }^{1}$ Veronica Lambert. 'Dublin City University, Dublin, Ireland; ${ }^{1}$ Institute of

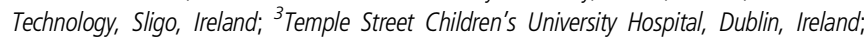
${ }^{4}$ Mater Misericordiae University Hospital, Dublin, Ireland; ${ }^{5}$ University of Leeds, Leeds, UK

\subsection{6/archdischild-2019-epa.586}

Aims Treatment of type 1 diabetes in adolescence requires input from parents to support the completion of complex multi-component self-management tasks in order to achieve optimal glycaemic control and maintain adolescent health and well-being. This study explored parents' experiences of learning about their child's type 1 diabetes including their initial reactions to the diagnosis and changes in their perspectives over time, in addition to parents' views of negotiating self-management responsibilities with their adolescent children.

Methods Qualitative interviews were conducted with 32 parents (24 mothers, 8 fathers) of adolescents, aged 11 to 17 years, living with type 1 diabetes to explore parents' experiences of learning about their child's type 1 diabetes and negotiating self-management responsibilities with them. Parents were recruited through two national child and adolescent diabetes and endocrine clinics and online advertisement through a national diabetes advocacy organisation. Interviews were transcribed verbatim and thematically analysed.

Findings Parents discussed their initial reactions to their child's type 1 diabetes diagnosis (e.g. upset, shock, worry). Parents identified the sources of support which they relied on following their child's diagnosis, including ongoing support from the diabetes team at their child's hospital and 